\title{
Detection and Separation of Multi-component Radar Emitter Signal
}

\author{
Xiaofeng Wang ${ }^{1, a}$, Guoyi Zhang ${ }^{2, b}$ and Lijun $\mathrm{Qi}^{3, \mathrm{c}}$ \\ ${ }^{1}$ Dept. Electronic Engineering, Naval Aeronautical and Astronautical University, Yantai 264001, China; \\ ${ }^{2}$ Dept. Electronic Countermeasure, Aviation University of Air Force, Changchun 130022, China. \\ ${ }^{3}$ Flight Research Institute, Aviation University of Air Force, Changchun 130022, China. \\ awxf870516@126.com, b'zhanggy1211@163.com, ${ }^{\mathrm{b}} 252636257 @ q q . c o m$
}

Keywords: signal detection, multi-component signal, time-varying filtering, support vector machine.

\begin{abstract}
The problem of multi-component radar emitter signal processing is studied, an effective method based on time-varying filtering is proposed to detect and separate the multi-component signal in radar reconnaissance occasion. Each signal component in the time-frequency plane is detected by the region growing method which is an image segmentation algorithm. Firstly, the linear frequency modulation (LFM) components in the multi-component signal are separated by fractional Fourier transform (FRFT). And then, the components which don't interact in the time or frequency domains are filtered. Finally, the components which interact in the time-frequency domains are separated by the support vector machine (SVM). Simulation results show that the proposed method can detect and separate multi-component signal effectively.
\end{abstract}

\section{Introduction}

Facing the increasingly complex electromagnetic environment, the wideband receiver intercepts more radar emitter signals in radar reconnaissance system. These emitter signals overlap in the time domain, which forms multi-component signal [1]. Detection and separation of multi-component radar emitter signals have become an urgent problem to be solved in the current electronic surveillance system. If the multi-component signal is processed as a single component signal, it will cause serious error for recognition and parameter extraction. Therefore, the multi-component signal should be detected firstly, followed by separation of each individual component. For the analysis of multi-component signal, the literature [2-4] study multi-component signal which consists of linear frequency modulation (LFM) signals, but the reconnaissance system often receives multiple types of radar signals actually, which means that only research of LFM signals does not have universality. Meanwhile, fractional Fourier transform (FRFT) in literature [2], Hough transform in literature [3], Radon transform in literature [4] all have no capability of time positioning, which are unable to determine the start time of the signal. The image processing techniques for processing multi-component signal is used in literature [3], but it is limited to the comparison of time-frequency image.

To solve the problems above, this paper uses a linear time-frequency transform for the detection of multi-component signal, and image processing for positioning of each component, a time-varying filter for separation of signals. Experimental results show that the proposed method can accurately detect the time and frequency range of each component of the signal and each component can be effectively separated with a strong anti-noise performance.

\section{Detection and Positioning of multi-component signal}

Detection of multi-component signal. Multi-component signal couples not only in the time domain coupling, but also couples in the frequency domain. The traditional analysis method in domain or frequency domain is difficult to conduct a valid analysis. Time-frequency analysis [6] can be used to characterize the two-dimensional distribution of time and frequency which provides a good idea for analysis of multi-component signal. In the time-frequency analysis methods, STFT is a linear 
time-frequency transform. The STFT of multi-component signal is the sum STFT of the each individual component, so this paper uses STFT to detect multi-component signal. STFT is defined as

$$
\operatorname{STFT}_{x}(t, w)=\int_{-\infty}^{\infty} x(\tau) w(\tau-t) e^{-\mathrm{jw} \tau} d \tau
$$

Where $w(t)$ is the window function. STFT obtain local frequency information via the sliding window in the time domain. The STFT of multi-component signal can be represented as

$$
\begin{aligned}
\operatorname{STFT}_{x}(t, w) & =\int_{-\infty}^{\infty} \sum_{i=1}^{L} s_{i}\left(t-T_{a i}\right) w(\tau-t) e^{-j w \tau} d \tau+\int_{-\infty}^{\infty} n(t) w(\tau-t) e^{-j w \tau} d \tau \\
& =\sum_{i=1}^{L} \operatorname{STFT}_{s_{i}}\left(t-T_{a i}, w\right)+\operatorname{STFT}_{n}(t, w)
\end{aligned}
$$

Formula (2) shows that each component has respective time-frequency area in the time-frequency domain, and each component can be separated one by one in the time-frequency domain.

Positioning of the each component in time-frequency distribution. In order to obtain the time and frequency range information for each component, providing the basis for the separation of signals, time-frequency positioning of each component is in need. While the time-frequency distribution of each component can be regarded as the foreground image, noise can be regarded as background image because it is uniformly distributed in the time-frequency distribution. Each point in time-frequency distribution can correspond to each pixel in the image, while the time-frequency amplitude corresponds to the gray value of image.

Region growing method [7] is commonly used in image segmentation, so the paper used image segmentation to separate the foreground from background. The foreground is decomposed into each component which can represent the region. The basic principle of regional growing is to make pixels with similar characteristics collected. Firstly, a "seed" pixel should be found in the image as a starting point, and then the neighborhood of the "seed" pixel which have similar characteristics should be combined to the area of the "seed" pixel in accordance with a certain growth criteria, continuously developing new neighborhood until it meets the stopping condition to complete the growth of a region.

Detection of linear time-frequency ridge and coupling. In order to facilitate the subsequent separation of multi-component signal, linear time-frequency ridge detection should be made, which determines whether it is LFM signal. Provided that $D_{i}$ is the $i$ th growing areas of component signal, searching the time-frequency ridge in the area of $D_{i}$, namely the maximum pixel value of the corresponding frequency at each time point. The $i$ th time-frequency ridge of component signal can be represented as

$$
\hat{f}_{i}(n)=\frac{1}{N} \arg \left[\max _{k \in D_{i}}|\operatorname{STFT}(n, k)|\right]
$$

Fitting $\hat{f}_{i}(n)$ linearly, when the fitting error is less than the threshold, it is determined as LFM signal, otherwise determined as other signals.

\section{Separation of multi-component signal}

Time-varying filtering based on FRFT. In order to avoid the influence of LFM signals to the separation of other signals, LFM signals are separated firstly using the time-varying filter. While non-time-frequency coupling components are then filtered, the time-frequency coupling components are finally separated. FRFT[3] is selected as time-varying filter to make the time-frequency plane rotate to a proper angle, separating the signal by constructing a filter, then inverse FRFT transforms the signal to the time domain. FRFT filter[8] can be expressed as

$$
r(t)=F^{-p}\left\{F^{p}[x(t)] H(u)\right\}
$$


Where $r(t)$ is the time-domain signal of separation, $F^{p}[\cdot]$ is the FRFT, $H(u)$ is the filter function. For the Separation of LFM signals, $H(u)$ may be designed as a bandpass filter with selecting an appropriate bandwidth, which can suppress some noise.

Determination of sorting line. The sorting line of LFM signals can be directly obtained from the time-frequency ridge, while time-frequency coupling components need to get the sorting lines with support vector machine[9,10] (SVM). Located sample set is $D=\left(x_{i}, y_{i}\right), i=1,2, \cdots, M, M$ is the total points of time-frequency ridge, $x_{i}$ is the point position of the ridge, $y_{i}$ is a flag of components, $y_{i} \in\{1,-1\}, 1$ or -1 represents the component is at the time-frequency ridge or not. SVM classifier training set is the points included by $D$. The goal is to find an optimal sorting line to distinguish the two regions, which can not only make correct classification, but also can make the class interval maximized. Sorting line is defined as

$$
f(x)=w^{\mathbf{T}} x+b
$$

Making the above formula normalized, it should satisfy that $y_{i}\left(w^{\mathrm{T}} x_{i}+b\right)-1 \geq 0, i=1,2, \cdots, M$ to make correct classification of all samples.

\section{Simulation}

In order to verify the effectiveness of the method, five components of the simulated radar emitter signal is used. The signal is made up with pulse of different length and continuous wave, namely LFM, non-linear frequency modulation (NLFM) and sinusoidal frequency modulated continuous wave (SFMCW) signal. The parameters of each component are shown in table 1 . Among them, the sampling frequency is set to $400 \mathrm{MHz}$, the size of FFT in STFT is 512 points, $\alpha$ is $0.5, \lambda$ is 3 . The noise is variance 1 , zero-mean Gaussian white noise. The amplitude of each component is determined by the SNR.

Table 1 Parameters of each component

\begin{tabular}{ccccc}
\hline Component & Modulation type & Frequency range/MHz & Time range/ $\mu \mathrm{s}$ & $\mathrm{SNR} / \mathrm{dB}$ \\
\hline 1 & LFM & $140 \sim 180$ & $0 \sim 20$ & 0 \\
2 & NLFM & $60 \sim 140$ & $2 \sim 8$ & 0 \\
3 & NLFM & $60 \sim 140$ & $7 \sim 10$ & 0 \\
4 & LFM & $140 \sim 200$ & $12 \sim 16$ & 0 \\
5 & SFMCW & $40 \sim 48$ & $0 \sim 20$ & 0 \\
\hline
\end{tabular}

When no noise is added (the ratio of signal amplitude has been determined), the waveforms of multi-component signal in the time domain and frequency domain is shown in figure 1(a) (b). It can be seen that there is a serious aliasing in the time domain and frequency domain, which is unable to effectively detect and separate signal. From figure 1(c) (d), we can see that STFT can clearly show the location of each component, and a serious cross-term in WVD. Therefore, STFT is better suited to handle multi-component signal than nonlinear time-frequency transform.

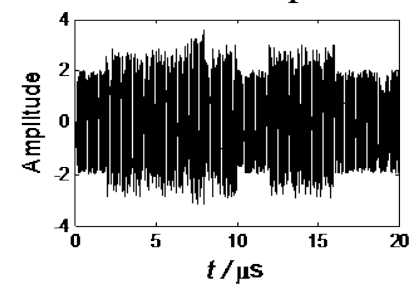

(a) time domain

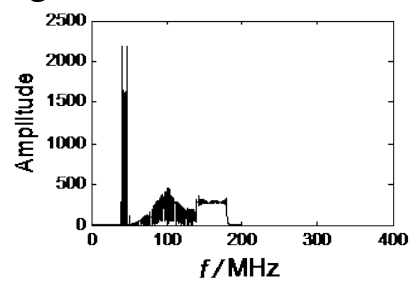

(b) frequency domain

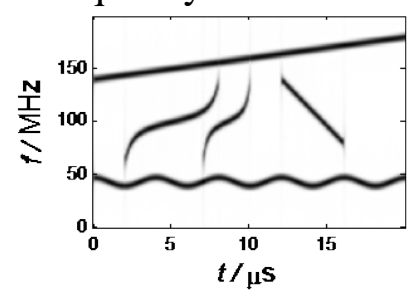

(c) STFT

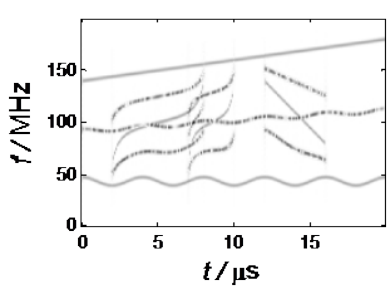

(d) WVD

Figure 1 Feature of multi-component signal without noise

In the case of table 1, the STFT of multi-component signal is shown in figure 2. As it can be seen, the influence of noise on each component is small. Figure 3 is a view of a ridge extracted from region growing, preferably reflecting the character of each component. Table 2 shows the detection results of multi-component signal. As it can be seen from table 2, the proposed method can accurately detect the time and frequency range of each component of the signal. In the case of no noise, the detection 
accuracy of the pulse width and the average bandwidth is greater than 95\%. At the average SNR of $0 \mathrm{~dB}$, the proposed method can still obtain high detection accuracy, with the detection accuracy of the pulse width and the average bandwidth greater than $90 \%$, resolving the problem of detection of multi-component signal.

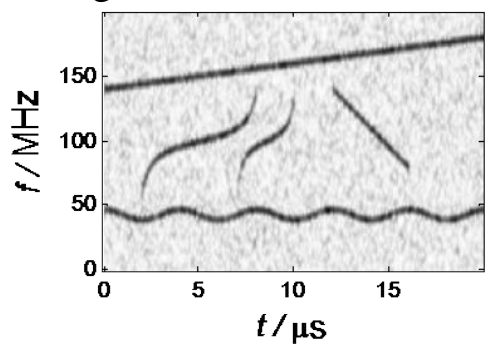

Figure 2 STFT with noise

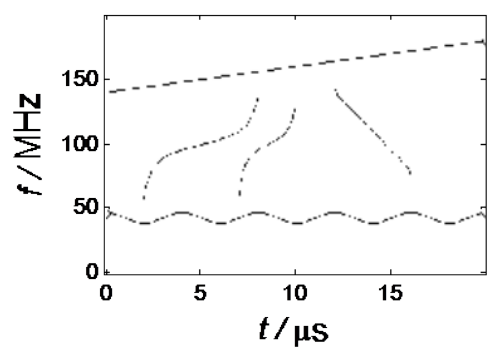

Figure 3 Time-frequency ridge

Table 2 Detection results of each component

\begin{tabular}{ccccc}
\hline \multirow{2}{*}{ Component } & \multicolumn{2}{c}{ Time range $/ \mu \mathrm{s}$} & \multicolumn{2}{c}{ Frequency range $/ \mathrm{MHz}$} \\
\cline { 2 - 5 } & Without noise & Average SNR=0dB & Without noise & Average SNR=0dB \\
\hline 1 & $0.01 \sim 19.98$ & $0.12 \sim 19.94$ & $140.6 \sim 179.7$ & $141.4 \sim 178.1$ \\
2 & $2.02 \sim 7.97$ & $2.08 \sim 7.92$ & $60.2 \sim 139.8$ & $62.6 \sim 138.2$ \\
3 & $7.01 \sim 9.97$ & $7.05 \sim 9.91$ & $60.2 \sim 139.8$ & $63.4 \sim 137.4$ \\
4 & $12.01 \sim 15.98$ & $12.12 \sim 15.92$ & $140.6 \sim 199.2$ & $142.2 \sim 198.4$ \\
5 & $0.02 \sim 19.98$ & $0.11 \sim 19.91$ & $40.6 \sim 47.7$ & $41.4 \sim 47.7$ \\
\hline
\end{tabular}

\section{Summary}

This paper proposes a new method for detection and separation of multi-component radar emitter signals. The method uses STFT to detect multi-component signal in time-frequency domain, using image segmentation techniques to position each component, using FRFT filtering to separate the various components in the time-frequency domain. Simulation results show the proposed method has better ability for detection and separation of multi-component signal. However, the paper can not effectively process the case that signals overlap or not be separated linearly in time-frequency plane, which requires further study.

\section{References}

[1] Rong Hai-na, Zhang Ge-xiang, Jin Wei-dong. Novel detection method for multi-component radar emitter signal[J]. Systems Engineering and Electronic, 2009,31(9): 2096-2010.

[2] Shao Ya-yong, Zhu Xiao-song. Separation and Detection of Signals by Fractional Fourier Transform Based on Cluste Analysis[J]. Telecommunication Engineering, 2012,52(2): 180-185.

[3] Li Nan, Qu Chang-wen, Ping Dian-fa, et al. Multi-components LFM signal recognition based on time-frequencyreassignment and Wigner-Hough transform[J]. Aerospace Electronic Warfare, 2009,25(6): 33-36.

[4] Zhang Bu-yun, Liu Ai-fang, Zhu Xiao-hua, et al. Multicomponent LFM Signal Detection and Parameter Estimation Based on Radon-STFT[J]. Journal of Detection \& Control, 2003,25(3): 30-33.

[5] Zou Xing-wen, Zhang Ge-xiang. Time-frequency analysis of radar emitter signals based on image processing technology[J]. JOURNAL OF CIRCUITS AND SYSTEMS, 2009, 14(3): 135-140.

[6] Zhang Xian-da, Bao Zheng. Non-stationary signal analysis and processing [M]. National Defence Industry Press, 1998.

[7] Yao Min. Digital image processing[M]. Machine-building Industry Press, 2006.

[8] Yan Ge, Liu Kai-hua, Luo Peng, et al. Fractional Fourier transform and novel time-frequency filter design[J]. JOURNAL OF HARBIN INSTITUTE OF TECHNOLOGY, 2012,44(9): 138-143. 
[9] Zhang Xue-gong. Introduction to statistical learning theory and support vector machines[J]. ACTA AUTOMATICA SINICA, 2000, 26(1): 36-46.

[10] Khan N A, Taj I A, Jaffri M N. Cross-term elimination in Wigner distribution based on 2D signal processing techniques [J]. Signal Processing, 2011,91(1): 590-599. 